\section{Competitive elimination of neuromuscular synapses}

SIR-Callaway et al. ${ }^{1}$ recently concluded that inactive motor neurons have an advantage over active motor neurons during neuromuscular synapse elimination in neonatal rabbit skeletal muscle. It is important to draw attention to certain limitations in their methodology and to exercise some caution in the interpretation of the data.

First, Callaway et al. report data obtained using only a single, indirect measure of innervation: twitch contractions of isolated skeletal muscles in response to stimulation of individual motor axons. It is well known that twitch tension measurements can overestimate both the amount of polyneuronal innervation and motor unit size, even though extreme precautions may be taken to ensure that the recordings are isometric ${ }^{2}$. Tetanic tension measurements are more reliable because time is given during a tetanus for series compliance in muscle to be saturated. Corroborative measurements, using intracellular recording or measurement of motor unit size using selective glycogen depletion of motor units, and histology ${ }^{3}$ are also necessary to support the contention.

Second, Callaway et al. base their conclusions on differences in the sizes of the twitch tensions produced only by the inactive motor units. There are two potential problems with interpretation here that could be addressed using direct methods. One is that, under some conditions, chronically paralysed neuromuscular junctions respond repetitively to single shock stimuli to the nerve, leading to summation of twitch tension, and therefore to spurious overestimates of motor unit size. This has been demonstrated using intracellular recording ${ }^{4}$. A second problem is that synapse elimination is delayed in muscle fibres which become innervated only by convergent terminals from separate tetrodotoxin-blocked motor neurons. At any particular stage in the developmental process this would produce larger than normal inactive motor units, but no difference in the size of active units in the same muscles, as Callaway et al. actually report.

Finally, Callaway et al. make an important, additional observation which should also be confirmed using direct methods: the measurements of tension overlap indicate that some muscle fibres become innervated exclusively by inactive motor axons. This does not mean that inactive axons must have had a competitive advantage. That conclusion could only be made if there had been a corresponding decrease in the mean size of the active motor units in the muscles. A simpler explanation is that factors other than differences in activity contribute to the selective stabilization of terminals; indeed it would be surprising if it were not so.

That muscle paralysis or stimulation influences the overall rate of synapse elimination is generally accepted $^{5-8}$. In relation to synaptic competition however, a cautious conclusion, based on the study of Callaway et al. and a complementary one by Ridge and Betz ${ }^{9}$, is that it is difficult to demonstrate a clear effect of differences in motor neuron activity during synapse elimination in immature skeletal muscle. Furthermore, such differences, if they occur, are small and of uncertain functional significance for the maturation of motor innervation patterns. Even during nerve regeneration, where the effects of inactivity during competition seem clear cut ${ }^{4,10}$, differences in motor neuron activity cannot be an overriding influence in the interactions between motor neurons during synapse formation and elimination.

\section{R. R. RIBCHESTER}

Department of Physiology,

University Medical School, Teviot Place, Edinburgh EH89AG, UK

1. Callaway, E.M., Soha, J.M. \& Van Essen, D.C. Nature 328, 422-426 (1987)

2. Brown, M.C. \& Matthews, P.B.C. J. Physiol., Lond. 151, $436-457(1960)$

. Jones, S.P. Ridge, R.M.A.P. \& Rowlerson, A. J. Physiol. Lond. 386, 377-384 (1987)

4. Ribchester, R.R. \& Taxt, T.J. Physiol., Lond. 344, 89-111 (1983)

. Thompson, W.J., Kuffler, D.P. \& Jansen, J.K.S. Neuro science 4, 271-281 (1978)

6. Thompson, W. Nature 302, 614-616 (1983).

7. O'Brien, R.A.D., Östberg, A. \& Vrbovà, G. J. Physiol., Lond. 282, 571-582 (1978)

. Taxt, T. J. Physiol, Lond. 340, 175-194.

9. Ridge, R.M.A.P. \& Betz, W.J. J. Neurosci. 4, 2614-2620 (1984)

10. Ribchester, R.R. in Electrical stimulation and neuromus cular disorders (eds Nix, W. \& Vrbovà, G.) 53-63 (Springer, Heidelberg, 1986).

Callaway ET al. RePly - Ribchester raises four specific concerns about our experimental paradigm and the interpretation of our results. First, adequacy of twitch tension measurements. We did not use tetanic tension measurements, because preliminary experiments showed that this led to gradual irreversible tension fatigue in our in vitro preparation. The key issue is not whether twitch tensions are less accurate than tetanic tensions as a measure of absolute motor unit size but whether they provide a reliable measure of changes in motor unit size in normal and experimental muscles. We agree that lack of perfect isometric recording conditions means twitch tensions tend to overestimate absolute motor unit size ${ }^{1}$, but the recording conditions are similar for all motor units from a given muscle. Twitch tensions therefore provide a reasonable indication of the relative size of different units in the same muscle.

We emphasize that our primary assay compared the relative tensions of motor unit populations from different spinal roots in the same animal. This withinanimal normalization procedure minimized the sensitivity of our analysis to any systematic tendency of twitch tensions to overestimate the precentage of muscle fibres in a motor unit. We would welcome any corroborative measurements using other procedures but we contend that the high statistical significance of our major findings demonstrates the sensitivity and reliability of our paradigm.

Second, repetitive nerve firing. Our estimates of twitch tension for inactive motor units would have been systematically overestimated if there was repetitive nerve firing in this subpopulation of motor axons. Inactivity-induced repetitive firing has been reported ${ }^{2}$ but only where much of the muscle was inactive; in these cases motor-unit twitch rise times were significantly slowed. Our paradigm was designed to maintain activity over nearly all the muscle and thus should not have been subject to this effect. In support, we found that the average rise time of motor units from tetrodotoxin (TTX)-inactivated roots was within 2 per cent of that for motor units from active roots in the same animals and for those of control and normal animals (fast and slow motor-unit rise times analysed separately).

Third, delayed synapse elimination. We presented evidence against a delay in synapse elimination at end-plates jointly innervated by active and inactive inputs. Ribchester suggests instead that the delay might have been restricted to end-plates that had lost their final active input but still received multiple inputs from inactive motor neurons. Such fibres should have been rare in our experiments and are unlikely to have contributed signifcantly to the larger size of inactive motor units. We have recently obtained more direct evidence against this possibility by allowing 5-7 days of recovery after 4-5 days of TTX inactivation. The previously inactivated motor units remain significantly larger than their active counterparts in control animals, by an amount comparable to that found in the short-term experiments (in preparation). Moreover, analysis of silver-stained end plates confirms that there is no significant degree of polyinnervation in these muscles. As well as arguing strongly against the delay hypothesis, these observations provide further evidence against the concern about repetitive firing, because the preparation had 5-7 days of presumably normal activity to recover from any side effects of inactivity.

Finally, decrease in size of active motor units. If inactive motor units have an advantage, the active motor units against which they compete should indeed have become smaller than normal. The magnitude of the expected difference is 